\title{
Experimental road signs in a simulated environment research programme - experiment procedures and assumptions
}

\author{
Mikołaj Kruszewski ${ }^{1,{ }^{*}}$, Monika Ucińska $^{1}$, Ewa Smoczyńska $^{1}$, Paweł Budziszewski ${ }^{2}$, and \\ Andrzej Grabowski ${ }^{2}$ \\ ${ }^{1}$ Motor Transport Institute, Warsaw, Poland \\ ${ }^{2}$ Central Institute for Labour Protection - National Research Institute, Warsaw, Poland
}

\begin{abstract}
The application of experimental signs on public roads may cause some risks due to driver misunderstanding or misinterpretation, especially when seen for the first time. To minimize this risk, driver reaction and sign understanding can be tested in a safe and relatively cheap simulation-based environment. The consortium running the project "Experimental road marking and its effect on road user behaviour" suggested a similar methodology. The project included a simulation-based research program. The consortium proposed a set of experimental signs which have a strong potential for improving road safety and traffic conditions. The paper outlines the simulation-based research programme involving experimental signs and a proposed experiment procedure. Simulation scenarios and procedures were selected to achieve the required message and goals of implementation. In addition, an analysis is proposed of the factors and indicators of each sign and its effects.
\end{abstract}

\section{Introduction}

Experimental signs are generally used to draw the driver's attention to a new problem or to restrict a certain behaviour. Because experimental signs are not commonly used, drivers may be faced with a sign for the first time in their life. Therefore, experimental signs on public roads may cause some risks due to driver misunderstanding or misinterpretation, affect traffic flow and lead to dangerous situations on the road. To minimize this risk and understand its influence, a simulated driving environment is used before the new signs are deployed in a real road environment. The consortium running the "Experimental road marking and its effect on road user behaviour" project, suggested a similar methodology. In the project, simulationbased experiments for the assessment of experimental signs will be introduced.

This approach was inspired by the promising results of other research teams that used simulation environments to assess how road signs influence the driver. Research conducted by Charlton [1], Rosey and Auberlet [2] or Ding et al. [3] shows that a driving simulation environment is suitable for conducting such research. Using the simulator we can evaluate driver behaviour in a safe environment and see whether the objectives underlying the establishment of a sign will be achieved. The research and the authors' earlier work were the

Corresponding author: mikolaj.kruszewski@its.waw.pl 
basis for proposing such a methodology. The procedures and assumptions of the proposed research are described in the chapters that follow.

\subsection{Experimental signs chosen for the simulation-based survey}

During the first phase of the project members of the Project consortium selected several experimental signs that should be the subject of the survey during the project. These signs included both horizontal signing and markings and vertical signs. Based on literature review and research results of other international research teams, the selected signs were tested in a simulation-based environment.

The following sign types were selected:

- horizontal markings which are the optical means of speed reduction at entries to intersections, junctions and ramp lanes

- horizontal curve markings with additional warning signs

- horizontal markings for speed reduction

- markings indicating the need to keep a safe distance

- extension of non-standard horizontal curve markings.

Some of the above signs are tested based on good practices and implementation models from other counties. The simulation-based experiment is in this case conducted to verify the influence of the sign on Polish drivers. Other signs, however, have different models of implementation in different countries. Especially in the case of vertical signs, a similar meaning of the sign could be shown using a different pictogram design in different countries. In such cases, the consortium agreed to perform survey-based research, to choose the most understandable sign pictogram.

\subsection{Survey-based research to choose sign pictograms}

Selecting the most understandable sign pictogram is one of the key elements when preparing for a simulation experiment. The drivers who will take part in the study will see these signs for the very first time. The meaning of the sign should be clear, and its comprehension should not influence their driving performance.

Based on literature review, the open-ended survey method based on ISO 9186-1 [4] was proposed. The ISO 9186-1 standard is dedicated to testing non-verbal presentation of information in public areas and specifies methods for assessing the comprehensibility of graphical symbols [4]. The method is used for evaluating the share of people who can correctly understand the meaning of a symbol or sign.

At least three designs of signs for each of the meanings were used in the survey. The designs were prepared according to ISO 7010 [5], dedicated to the preparation of safety signs in public areas, and in accordance with national law on graphic symbols to be used in road signs in Poland [6].

For each sign two questions are provided:

- What action should you take in response to this symbol?

- What do you think is the meaning of this symbol?

The respondent should answer both questions with a few words describing what they thought first when they saw the symbol. Figure 1 shows an example from the questionnaire. 


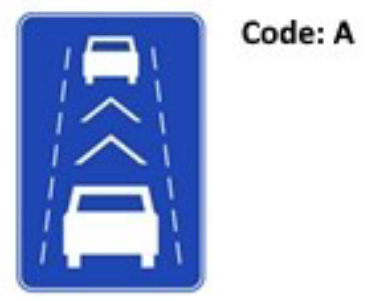

\begin{abstract}
This road sign is a vertical sign placed along the road. You can see it on the rural highways, motorwsys and expressways.

Deseribe in a few words what you should do when you see this sign?
\end{abstract}

Describe in a few words what you think this rosd sign means?

Fig. 1. Example of the survey showing a proposed sign design - horizontal road markings indicating the need to keep a safe distance to the vehicle in front.

Every response was categorized into one of five possible categories depending on the degree of correctness. The survey is planned for at least 300 respondents. The results should suggest which of the proposed sign designs is easiest to understand. The signs were used in simulation-based experiment.

\title{
2 Experiment procedures
}

The simulation-based experiment was conducted in accordance with the internal rules and good practices of the Driving Simulation Laboratory of the Motor Transport Institute. The simulation-based experiments need to comply with the safety procedures that include e.g. simulator sickness prevention rules or adaptation period. The experiment procedure will be in line with these rules providing suitable breaks for the participants and a corresponding sequence of tests. The whole experiment will be divided into relatively short simulation rides depending on the final number of road situations to be tested. Figure 2 shows the universal procedure scheme used in Simulation Laboratory.

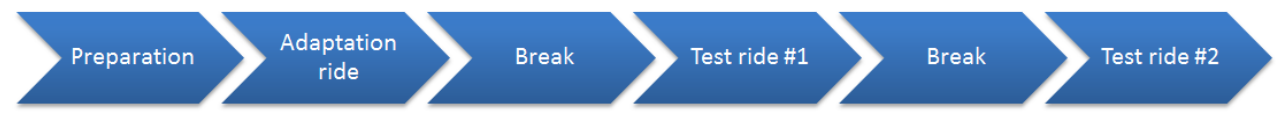

Fig. 2. Example of a Simulation Laboratory research procedure used for experiments divided into two test rides.

The procedure applied in Simulation Laboratory helps to control the occurrence of simulation sickness and takes into account the necessity for the driver to adapt their skills to the specifics of driving a car in a simulation environment. A similar procedure was implemented successfully in a previous research e.g. EYEVID [7] and GEMS [8,9].

\subsection{Apparatus}

For the simulation-based experiment the project consortium plans to use two research tools. First, the AS1200-6 simulator. It is a high fidelity simulator built using the Opel Astra 4 cabin situated on a motion platform with 6 degrees of freedom of movement. The simulation environment is The SimWorld provided by AutoSim. The simulator records all data regarding the use of steering equipment. 

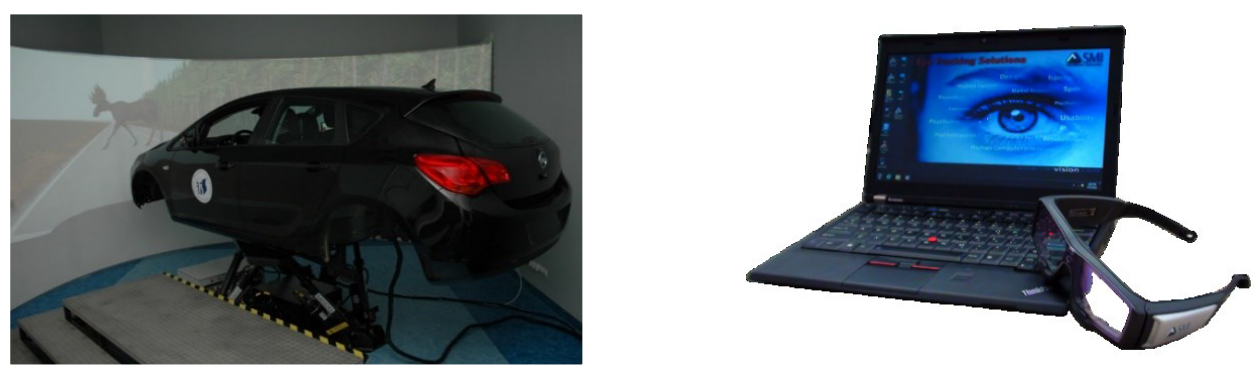

Fig. 3. Research tools intended to be used in the experiments. Left: AS1200-6 simulator. Right: SMI Eyetracking Glasses 1.8 mobile eyetracker.

Due to the project scope, the consortium decided to use the eyetracker to register the visual activity of the participants. The mobile eyetracker EyeTracking Glasses 1.8 of the SensoMotoric Instruments will be used. This tool should provide information about the time spent on sign observation, an indicator of a possible driver distraction.

\subsection{Survey assumptions}

Literature review provided information about the most popular parameters analysed in research regarding the influence of road infrastructure on the driver. Most effects are observed in lateral or longitudinal steering behaviour depending on the type of sign used in research. The following parameters were indicated to be recorded during experiments:

- Lane excursion

- Lateral behaviour

- Headway

- Time to collision

- Gas pedal steering

- Steering wheel reversal rate

- Speed.

All the above parameters would be registered and analysed during simulation-based research. For each parameter the analysis of the behaviour will be conducted on the section before the sign, in the area of the sign's impact and on the section after the sign.

For each of the signs a suitable road situation will be presented. The driver will drive in two similar road situations, one with the experimental sign, and one without it or with the standard road sign. Figure 4 shows an example of an experimental road situation.
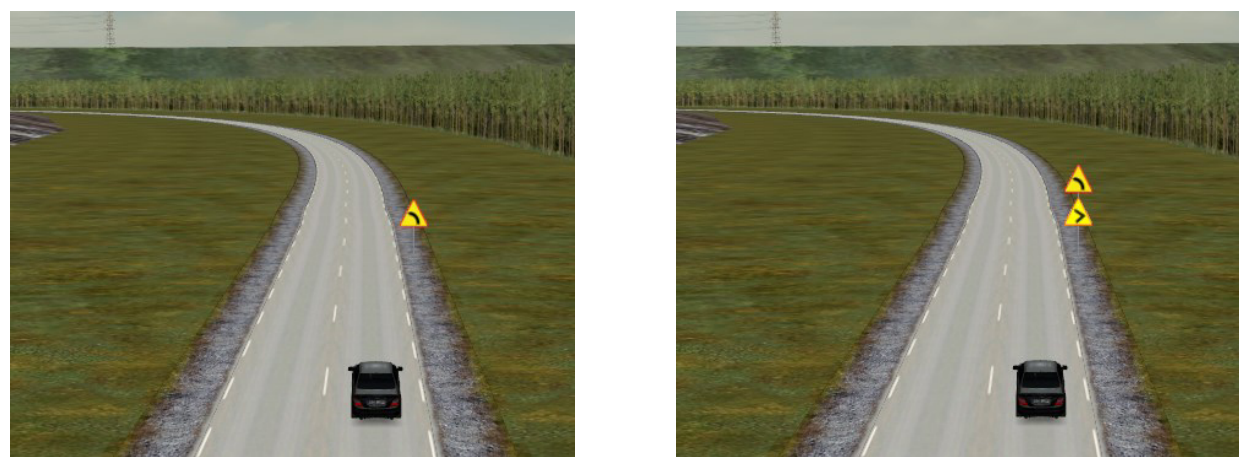

Fig. 4. Example of an experimental road situation. Left: road curve with classical signing. Right: road curve with an experimental sign supplementing the classical sign. 
The effect of the experimental sign will be compared to the standard sign in the same road situation. The comparison should suggest whether the expected results of the experimental sign have been achieved and whether the sign does not induce inadequate or dangerous behaviour. At least 50 drivers (different age, sex, and experience) are planned to participate in the experiment.

\subsection{Control of simulator sickness occurrence}

Simulation sickness control is a common problem for all research conducted in simulation environments. As indicated by Cobb et al. [10] simulator sickness is a problem that may:

- affect research results,

- cause that some participants may not be able to complete the tests carried out in the driving simulator.

To evaluate the occurrence of symptoms of simulator sickness the survey tool called RSSQ (Revised Simulation Sickness Questionnaire) was introduced. The RSSQ used in Simulation Laboratory of the Motor Transport Institute is a direct implementation of RSSQ developed by Kennedy et al. [11]. The survey includes 29 questions about the occurrence of the symptoms. Responses are evaluated in three categories of nausea, occulomotoric and disorientation symptoms.

The RSSQ is a post hoc method of simulation sickness evaluation. It helps to check how research results are influenced by helping to eliminate from the analysis the results of participants who may suffer significant inconvenience because of the symptoms. The results of these drivers are removed from the analysis.

Respondent's comfort (before driving simulator - adaptation)

The questionnaire aims to check what is your current comfort. Mark with a cross $(X)$ which of the sym ptoms given below refers to your feeling status. Only one answer per line is allowed.

\begin{tabular}{|c|l|l|l|l|l|}
\hline LP & \multicolumn{1}{|c|}{ Symptoms } & None & Slight & Moderate & Severe \\
\hline 1 & General discomfort & & & & \\
\hline 2 & Fatigue & & & & \\
\hline 3 & Tiredness & & & & \\
\hline 4 & Somnolence & & & & \\
\hline 5 & Headache & & & & \\
\hline 6 & Eyestrain & & & & \\
\hline 7 & Difficulty focusing & & & & \\
\hline 8 a & Increased salivation & & & & \\
\hline 8 b & Dry mouth & & & & \\
\hline 9 & Sweating & & & & \\
\hline 10 & Nausea & & & & \\
\hline 11 & Difficulty concentrating & & & \\
\hline 12 & Depression & & & & \\
\hline 13 & Disorientation & & & \\
\hline 14 & Blurred vision & & & \\
\hline 15 a & Daze at eyes opened & & & \\
\hline $15 b$ & Daze at eyes closed & & & \\
\hline 16 & Dizziness & & & & \\
\hline 17 & Flashes of memory & & & & \\
\hline 18 & General weakness & & & & \\
\hline 19 & Need to take a breath & & & \\
\hline 20 & Stomach discomfort & & & \\
\hline 21 & Loss of appetite & & & \\
\hline 22 & Increased appetite & & & \\
\hline 23 & Need to defecate & & & \\
\hline 24 & Sense of confusion & & & \\
\hline 25 & Feeling of bouncing from stomach & & & \\
\hline 26 & Vomiting & & & \\
\hline 27 & Others: ..................... & & & \\
\hline & & & & \\
\hline
\end{tabular}

Fig. 5. Revised Simulation Sickness Questionnaire. 


\section{Conclusions}

The process of implementing new road signs must take road safety as a priority. The influence of experimental signs on drivers depends on different contexts (e.g. cultural, organizational, legal, etc.) and needs to be carefully examined before the proposed signs are introduced on the roads. Surveys and a good class driving simulator seem to be the best solution for this purpose due to the controlled and repeatable simulation environment as well as precise driving data registered during the drive.

In the "Experimental road marking and its effect on road user behaviour" project a detailed research procedure was designed in order to deal with the presented topic. The method is based on the literature, international standards and good practices of members of the Project Consortium and provides a two-step procedure for choosing the best-fitting road signs. A simulation-based driving experiment, preceded with selecting the best signs evaluated in an online survey, will provide a sufficient amount of data to evaluate the change in driver's behaviour when faced with the new signs. This procedure is easy to implement and cost-efficient and could significantly simplify the process of road sign implementation, taking into account all accompanying factors.

\section{Acknowledgements}

The project "Experimental road marking and its effect on road user behaviour" is financed by the National Centre for Research and Development (NCBiR) and the General Directorate for National Roads and Motorways (GDDKiA), grant agreement DZP/RID-I-36/5/NCBR/2016.

\section{References}

1. S.G. Charlton, The role of attention in horizontal curves: A comparison of advance warning, delineation, and road marking treatments, Accid. Anal. Prev. 39 pp. 873-885 (2007)

2. F. Rosey, J.-M. Auberlet, Trajectory variability: Road geometry difficulty, Saf. Sci. 50 pp. 1818-1828 (2012)

3. H. Ding, X. Zhao, J. Rong, J. Ma, Experimental research on the effectiveness of speed reduction markings based on driving simulation: A case study, Accid. Anal. Prev. 60 pp. 211-218 (2013)

4. Graphical symbols - Test methods - Part 1: Method for testing comprehensibility. ISO Norm 9186-1 (2014)

5. Graphical symbols -- Safety colours and safety signs - Safety signs used in workplaces and public areas. ISO Norm 7010 (2011)

6. Regulation of the Ministers of Infrastructure and Internal Affairs and Administration of 31 July 2002 on road signs and signals. OJ 2002 no. 170 pos. 1393 (Poland, 2002)

7. M. Kruszewski, P. Razin, M. Niezgoda, E. Smoczyńska, T. Kamiński, Analiza efektów oddziaływania symulatora na powstawanie choroby symulatorowej $\mathrm{w}$ badaniach kierowców, Syst. Logistyczne Wojsk (in Publ. 44 (2016)

8. M. Niezgoda, T. Kamiński, M. Kruszewski, A. Tarnowski, Self-reported drivers' behavior: an application of DBQ in Poland, J. Kones Powertrain Transp. (2013)

9. M. Niezgoda, A. Tarnowski, M. Kruszewski, T. Kamiński, Towards testing auditoryvocal interfaces and detecting distraction while driving: A comparison of eyemovement measures in the assessment of cognitive workload, Transp. Res. Part F. 32 pp. 23-34 (2015) 
10. S. Cobb, S. Nichols, A. Ramsey, J. Wilson, Virtual reality-induced symptoms and effects (VRISE), Presence. 8(2) pp. 169-186 (1999)

11. R.S. Kennedy, N.E. Lane, K.S. Berbaum, M.G. Lilienthal, A simulator sickness questionnaire (SSQ): A new method for quantifying simulator sickness, Int. J. Aviat. Psychol. 3(3) pp. 203-220 (1993) 